\title{
The Temporal Relationship between Selected Mental Disorders and Substance-Related Disorders: A Nationwide Population-Based Cohort Study
}

\author{
Mu-Lin Chiu, ${ }^{1,2}$ Chi-Fung Cheng, ${ }^{2}$ Wen-Miin Liang,, ${ }^{2,3}$ Pen-Tang Lin,, \\ Trong-Neng Wu ${ }^{D},{ }^{5}$ and Chiu-Ying Chen (D) ${ }^{2,6}$ \\ ${ }^{1}$ National Institute of Infectious Diseases and Vaccinology, National Health Research Institutes, Zhunan, Taiwan
${ }^{2}$ Department of Public Health, China Medical University, Taichung, Taiwan
${ }^{3}$ Graduate Institute of Biostatistics, China Medical University, Taichung, Taiwan
${ }^{4}$ Psychiatric Department, Taichung Veterans General Hospital, Taichung, Taiwan
${ }^{5}$ Department of Healthcare Administration, Asia University, Taichung, Taiwan
${ }^{6}$ Graduate Institute of Clinical Medical Science, College of Medicine, China Medical University, Taichung, Taiwan
}

Correspondence should be addressed to Trong-Neng Wu; tnwu@asia.edu.tw and Chiu-Ying Chen; chence88@gmail.com

Received 8 February 2018; Accepted 19 August 2018; Published 4 October 2018

Academic Editor: Umberto Albert

Copyright (C) $2018 \mathrm{Mu}$-Lin Chiu et al. This is an open access article distributed under the Creative Commons Attribution License, which permits unrestricted use, distribution, and reproduction in any medium, provided the original work is properly cited.

\begin{abstract}
Introduction. Previous studies have examined the association between specific mental disorders, particularly mood and anxiety disorders, and substance-related disorders; but the temporal link between them remains unclear. This study aimed to examine whether individuals with specific mental disorders, including affective psychoses, neurotic disorders, schizophrenia, personality disorders, and adjustment reaction, have higher risks for subsequently developing substance-related disorders compared to those without. Methods. A large-scale study with longitudinal data was conducted using the Taiwan National Health Insurance Research Database (NHIRD) consisting of 2,000,118 patients' medical records from 2000 to 2009. A total of 124,423 people diagnosed with selected mental disorders and the same number of people without the diagnoses of the selected disorders were identified between January 1, 2001, and December 31, 2006, and followed up for the diagnoses of substance-related disorders till the end of 2009. We estimated the risk for subsequently developing substance-related disorders among patients with the selected mental disorders compared to those without by using Cox proportional hazard models. The cumulative incidence of substance-related disorders was calculated using the Kaplan-Meier method. Results. The risk for developing substance-related disorders in patients with selected mental disorders is about 5 times $(\mathrm{HR}=5.09,95 \% \mathrm{CI}: 4.74-5.48)$ higher than those without after adjusting for potential confounding variables. From the multivariate analyses of subsamples stratified by age, sex, and urban and income levels, we found all adjusted hazard ratios were significantly higher than 1.0, ranging from 2.12 (95\% CI: 1.72-2.62) to 14.55 (95\% CI: 7.89-26.83). For children and adolescents aged 10-19 years, those with specific mental disorders had 14.55-fold higher risk for developing substance-related disorders in later life compared to their counterparts. Furthermore, patients with personality disorders had the highest risk $(\mathrm{HR}=25.05)$. Conclusions. The earlier onset of the selected mental disorders is a potential risk for developing substancerelated disorders in later life, particularly for personality disorders. Health professionals should pay more attention to this at-risk population, especially to adolescents with mental disorders.
\end{abstract}

\section{Introduction}

Substance-related disorders (SRDs), comprised of substance use disorders (SUDs) and substance-induced disorders (SIDs), are disorders of intoxication, dependence, abuse, and substance withdrawal caused by various substances, both legal and illegal. According to the 2012 World Drug Report made by the United Nations Office on Drugs and Crime (UNODC), every year 2.3 million, 5.1 million, and 250,000 people die from alcohol, tobacco, and drug-related illnesses, 
respectively. Among the global population aged 15 years and older, the annual prevalence rate for alcohol use is $42 \%$, for tobacco use is $25 \%$, and for illicit drug use is 5\% [1]. The subsequent negative effects of these substance-related problems on population health and increasing economic and social burdens have become an important issue in global health $[2,3]$.

The coexistence of mental disorders with substance use or substance-related disorders has been well documented [4-7]. For example, the estimated U.S. population lifetime prevalence of comorbid alcohol and drug disorder for adults with a mental disorder was $29 \%$. Notably, the prevalence rates of comorbidity of any of mental disorders for adults with any addictive disorders (36.6\% for alcohol; $53 \%$ for drug disorders) were all higher than the aforementioned comorbidity rate [4].

Existing studies have further examined the temporal relationships between psychiatric disorders and substance disorders or substance use in understanding the etiology among the disorders, but the link was inconclusive. Some indicated that earlier substance use or SUDs could increase the risks for the subsequent onset of depressive, bipolar, and anxiety disorders [8-14], while others indicated contrasting results $[5,15-21]$. These contrasts may be due to the variations in the methodology such as study design, sample selection, and onset measurement and due to the limitations from small sample sizes or short follow-up periods across the studies. Most longitudinal studies with retrospective data encounter recall bias from self-report information [5, 9-16]. Several studies lack information on childhood and early adolescence [9-13]. Some studies are limited to small sample sizes [15, 22], while others with large sample sizes are not based on community populations or not nationally representative $[14,16,22]$. However, recent studies have suggested that an underlying susceptibility to mental disorders causes people to use substances to self-medicate the subthreshold symptoms of their incipient mental disorders, resulting in the full diagnosis of SUDs [21-23]. Hence, the temporal link between mental disorders and substance-related disorders requires clarification.

A body of literature indicated that mental problems occurring in childhood and adolescence could increase the risk for substance use and/or abuse in adulthood [2427]. In addition, among the empirical studies we found more research considering mood disorders, anxiety disorders, personality disorders, and conduct disorders as risk factors for SRDs than schizophrenia or other psychiatric diseases $[5,7,15,22,28]$. Therefore, the primary interest of our study is to examine the temporal relationship of the specific mental disorders including affective psychoses, neurotic disorders, personality disorders, and adjustment reaction as well as schizophrenia with the later onset of substance-related disorders by using data with follow-up medical records from a large population-based sample of more than two million individuals, which allowed us to ascertain the diseases' diagnoses and their temporal links. Previous studies have indicated that characteristics such as age [29-31], sex [32-35], education level [36], income and employment [37, 38], and socioeconomic status [39-44], as well as urban and rural status [45-48], all have effects on the rate of substance use or abuse. Therefore, we adjusted for these potential confounding variables to investigate the temporal pattern of the onset of the above-mentioned mental disorders prior to SRDs by adopting a random sampling strategy with matching the characteristics for those with the selected mental disorders in comparison to those without.

\section{Methods}

2.1. Data Source. We utilized a database of $2,000,118$ beneficiaries, randomly sampled from the registration data of all beneficiaries enrolled in the Taiwan National Health Insurance Program (TNHIP) in the year 2000. Individual medical records were followed up till the end of the year 2009, and available records could be traced back to the beginning of year 1995 of TNHIP, namely, the National Health Insurance Research Database (NHIRD). This is a nationally representative sample managed and provided by the Collaboration Center of Health Information Application (CCHIA) at the Ministry of Health and Welfare of Taiwan. The enrolled beneficiaries cover $99.9 \%$ of over 23 million people of Taiwan [49]. NHIRD is widely used because of its largescale and longitudinal advantages. As the database consists of de-identified secondary data for research purposes, this study was exempt from a full ethics review and instead only required a basic one approved by the Institutional Review Board at China Medical University Hospital.

2.2. Study Design. We conducted a retrospective cohort study and observed two cohorts, one with a selected mental disorder and one without, and traced their medical outcome of having been diagnosed with a substance-related disorder. The cohort of beneficiaries with selected mental disorders consisted of people diagnosed with schizophrenia, affective psychoses, neurotic disorders, personality disorders, or adjustment reaction in accordance with the International Classification of Diseases, Ninth Revision, Clinical Modification (ICD-9-CM) codes of 295.xx, 296.xx, 300.xx, 301.xx, or 309.xx, respectively, from January 1, 2001, to December 31, 2006. To increase the validity of clinical diagnoses, mental disorder classification was defined as having a history of at least three outpatient visits within one year or a one-time hospitalization. The first day a person is diagnosed with any one of the selected mental disorders identified from at most three ICD-9-CM codes (in addition to the principal diagnostic code, the secondary and tertiary diagnostic codes were considered if they existed) was defined as the index date. The cohort of the control group was randomly selected from those beneficiaries without selected mental disorders with matching by age ( \pm 3 years), sex, urban level, income level, and index date at a ratio of 1:1 (case:control) within the same period. The levels of urbanization were categorized according to the stratification of Taiwan Townships developed by Liu et al. [50]. Six income levels were categorized according to the insurance amount classified by the National Health Insurance Administration, Taiwan. Subjects who were diagnosed with substance-related disorders before the index date, on the 
index date, and one year after the index date were all excluded from our study.

The substance-related disorders (SRDs), including alcohol-induced mental disorders, drug-induced mental disorders, alcohol dependence syndrome, drug dependence, or nondependent abuse of drugs, were identified in accordance with the ICD-9-CM codes of 291.xx, 292.xx, 303.xx, 304.xx, or 305.xx, respectively. And we also defined substance-related disorders as having the diagnostic codes in at least three outpatient visits within one year or a onetime hospitalization from January 1, 2001, to the end of December 31, 2009. Finally, we obtained a total of 124,423 subjects with selected mental disorders and the same size of control subjects. Among the two cohorts, for each matched pair, the period of time after the index date was set as the entire observational period, and we followed up until the occurrence of substance-related disorders, death, drop out from the insurance system, or the end of 2009. Therefore, each subject was followed for at least 3 years.

2.3. Statistical Analysis. To determine whether specific mental disorders are the antecedents to substance-related disorders, the study estimated their hazard ratios (HRs) adjusted for age, sex, and urban and income levels using the Cox proportional hazard models. Furthermore, the model was also used to analyze subsamples stratified by age, sex, and urban and income levels to examine relationships across subpopulations. The Kaplan-Meier method was used to calculate the cumulative incidence of substance-related disorders in subjects with selected mental disorders and those without. The model was also used for obtaining the estimated risks of each subtype of SRDs for the selected mental disorders as compared to their counterparts by adjusting for age, sex, and urban and income levels. Before conducting the above analyses, the comparison of the baseline characteristics between two cohort subjects was made using a $\chi^{2}$ test for categorical variables. All data management and calculation of HR and 95\% Confidence Interval (CI) were performed using the SAS System (version 9.3; SAS Institute, Cary, NC). Results with a $\mathrm{p}$ value less than .05 were considered statistically significant.

\section{Results}

From the longitudinal health insurance database, this study identified 124,423 patients clinically diagnosed with selected mental disorders between 2001 and 2006. Simultaneously, this study randomly selected 124,423 people without selected mental disorders as the control cohort matched to each case's index date, age, sex, and urban and income levels after excluding subjects who had SRDs before, on, and one year after the index date. The mean follow-up duration was $6.19( \pm 1.75)$ years. Figure 1 shows the details of the process of selecting study subjects. Due to our matching process, the distributions of age, sex, urban level, and income level were similar at the baseline between the cohort subjects with selected mental disorders and those without. Among the subjects with the selected mental disorders, the majority of them were diagnosed with neurotic disorders (91.71\%), followed by affective psychoses (17.48\%), adjustment reaction (4.93\%), schizophrenia (2.65\%), and personality disorders $(1.37 \%)$ in descending order (Table 1), suggesting that neurotic disorders were the most prevalent comorbid diseases, followed by comorbid affective psychoses.

Table 2 shows the follow-up profiles for the two groups. The median duration from the index date of the diagnosis to the subsequent onset of SRDs in the mental disorder group was shorter than that in the control $(1.54<3.22$ years), and the difference in the mean duration was significant $(2.12<$ 3.28 years, $p<0.001)$. The proportions of the subsequent SRD cases and death during the follow-up period in the mental disorder group were more than that in the control $(3.41 \%>0.69 \%$ and $8.34 \%>6.9 \%$, respectively). The proportion of the loss of follow-up in both groups was similar and few $(0.17 \%$ and $0.16 \%)$. It is interesting to note that the major subtype of SRDs was the nondependent abuse of drugs in both the mental disorder group and the control group, followed by alcohol dependence syndrome $(1.75 \%>0.95 \%$; $0.47 \%>0.13 \%)$. Figure 2 displays the cumulative incidences of SRDs both in mental disorder and in control groups. The cumulative incidence of SRDs in the mental disorder group increased faster than that in the control group and reached 5 times higher by the end of year $2009(p<0.05)$.

Table 3 shows the result of the multivariable Cox proportional hazard regression model, an adjusted HR of 5.09 (95\% CI: 4.74-5.48), suggesting that the risk of subsequent onset of SRDs in persons with selected mental disorders is about five times higher than that in those without after controlling for age, sex, and urban and income levels. Across the different diagnoses of selected mental disorders, patients with personality disorders were at the highest risk $(\mathrm{HR}=25.05,95 \%$ $\mathrm{CI}=14.37-43.67)$, followed by schizophrenia (HR=11.17, 95\% $\mathrm{CI}=7.73-16.14)$, affective psychoses ( $\mathrm{HR}=10.99,95 \% \mathrm{CI}=9.36$ $12.90)$, adjustment reaction disorders $(\mathrm{HR}=9.67,95 \% \mathrm{CI}=$ 6.97-13.51), and neurotic disorders ( $\mathrm{HR}=4.84,95 \% \mathrm{CI}=4.48$ 5.23). Adjusted HRs for age, sex, and urban and income levels appear significant. Compared to the elderly age group $(\geqq 70$ years), all other age groups had higher risks, particularly the age groups in the 20-49-year range, where hazard ratios (HR) ranged from $5.61(95 \% \mathrm{CI}=4.48-5.95)$ to $7.92(95 \% \mathrm{CI}=6.89$ 9.11). People aged 30-39 had an almost 8-fold higher risk for developing SRDs than the elderly. Males had over a 4fold higher risk $(\mathrm{HR}=4.30 ; 95 \% \mathrm{CI}=4.04-4.57)$ than females. People living in the lowest urbanization areas (HR=1.30; 95\% $\mathrm{CI}=1.19-1.43$ ) showed the highest risk for developing SRDs. Compared to persons in the highest income level, those in every lower income level were at significantly higher risks for having SRDs. Individuals in the lowest income level ( $\leqq$ NT\$ 18,780 ) showed the highest HR.

Furthermore, when we analyzed subsamples stratified by age, sex, and urban and income levels by using multivariable Cox proportional hazard models (Table 4), we found the adjusted HRs were all significantly higher than 1.0, with hazard ratios ranging from 2.12 ( $95 \% \mathrm{CI}=1.72-2.62)$ to 14.55 (95\% CI=7.89-26.83), including age (10 to 70 years or more), gender (males and females), urban level (lowest to highest urbanization), and income level ( $\leqq 18,780$ to $\geqq 45,801)$. Among 


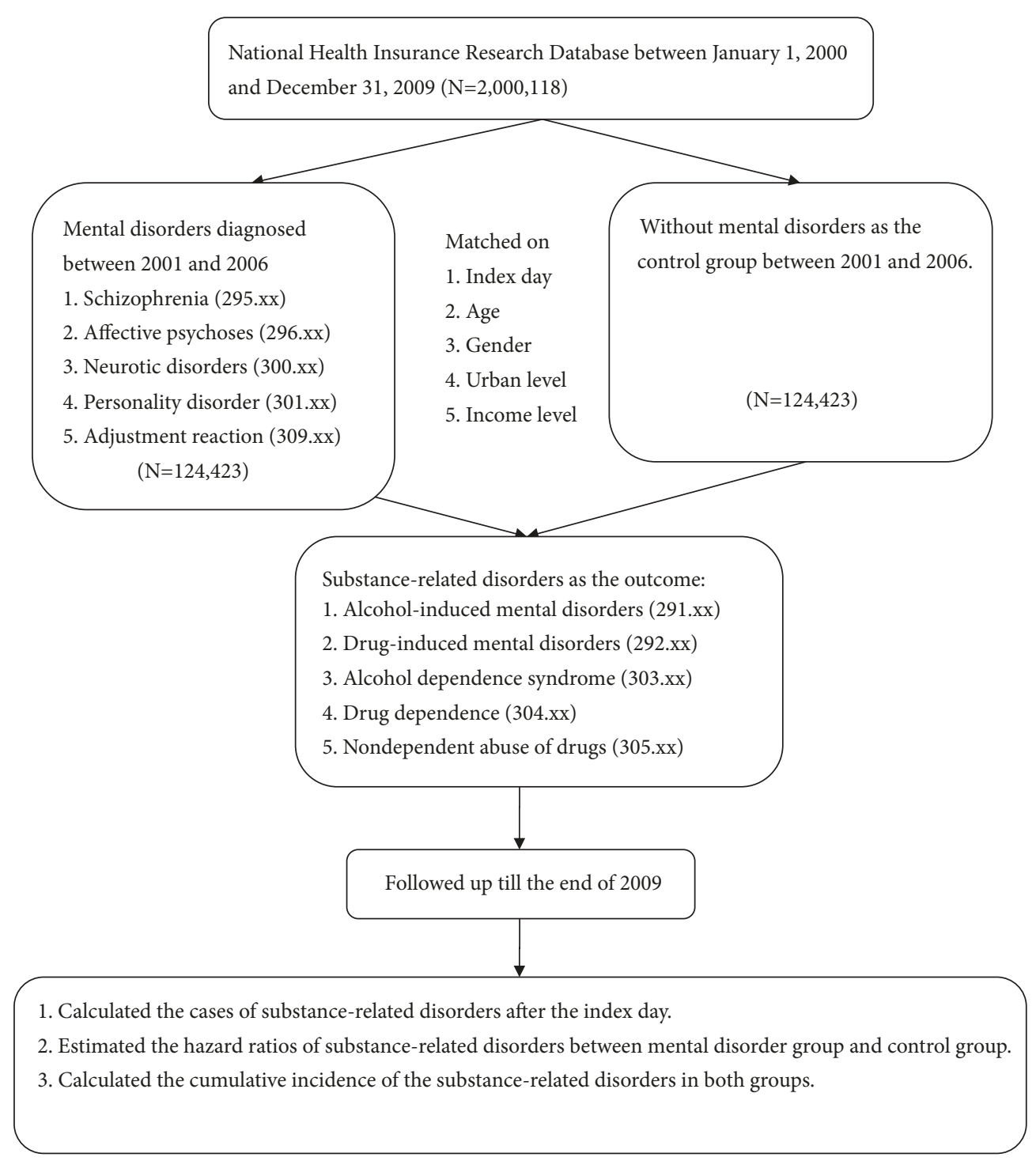

Figure 1: Study flow.

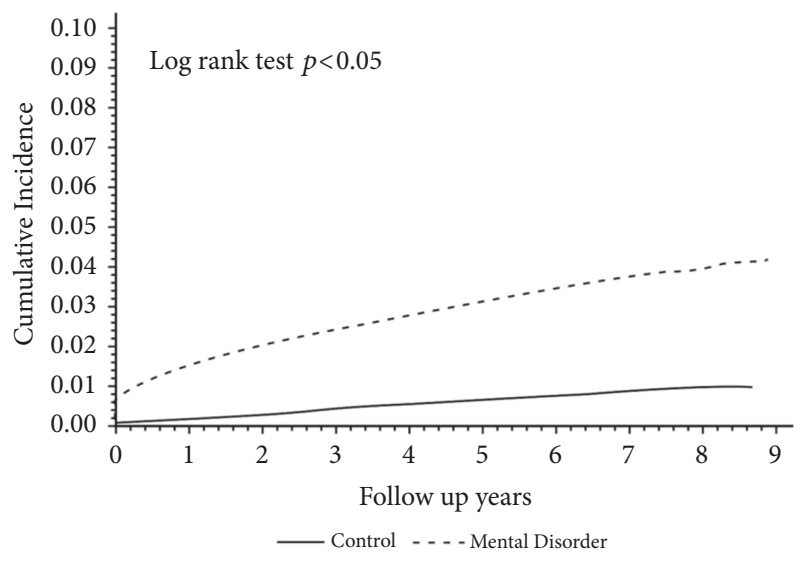

FIGURE 2: Cumulative incidence of substance-related disorders in patients with mental disorders compared to those without. the individuals aged 10-19, those with selected mental disorders had a 14.55-fold higher risk for subsequent SRDs than those without and, interestingly, the hazard ratio increased as age decreased. Males with selected mental disorders had around a 9 times $(\mathrm{HR}=8.68$; 95\% $\mathrm{CI}=7.35-10.26)$ higher risk for developing SRDs than those without. For people living in the urbanization levels above moderate, those with selected mental disorders would have around a 6 times higher risk for developing SRDs than those without. When stratified by income level, among individuals at the income level of NT\$ 28,801-36,300, those with selected mental disorders showed close to a 7 times higher risk $(\mathrm{HR}=6.62 ; 95 \% \mathrm{CI}$ : 4.54-9.66) for developing SRDs than those without.

Table 5 shows the estimated hazard ratios of subsequent onset of each subtype of substance-related disorders for each selected mental disorder while accounting for age, 
TABLE 1: The distribution of sociodemographic characteristics between mental illness and control cohorts and of the mental illness at baseline.

\begin{tabular}{|c|c|c|c|c|c|}
\hline & \multicolumn{2}{|c|}{$\begin{array}{l}\text { Mental Illness } \\
(\mathrm{N}=124,423)\end{array}$} & \multicolumn{2}{|c|}{$\begin{array}{c}\text { Control } \\
(\mathrm{N}=124,423)\end{array}$} & \multirow[t]{2}{*}{$P$-value } \\
\hline & $\mathrm{N}$ & $(\%)$ & $\mathrm{N}$ & (\%) & \\
\hline \multicolumn{6}{|l|}{ Disease category ${ }^{¥}$} \\
\hline Neurotic disorders & 114,114 & $(91.71 \%)$ & & & \\
\hline Affective psychoses & 21,754 & $(17.48 \%)$ & & & \\
\hline Adjustment reaction & 6,133 & $(4.93 \%)$ & & & \\
\hline Schizophrenia & 3,297 & $(2.65 \%)$ & & & \\
\hline Personality disorders & 1,710 & $(1.37 \%)$ & & & \\
\hline Age (years) & 48.84 & $(17.10)$ & 48.78 & $(17.11)$ & $0.436^{\dagger}$ \\
\hline 10-19 & 4964 & $(3.99 \%)$ & 5161 & $(4.15 \%)$ & 0.548 \\
\hline $20-29$ & 14632 & $(11.76 \%)$ & 14517 & $(11.67 \%)$ & \\
\hline $30-39$ & 20091 & $(16.15 \%)$ & 20106 & $(16.16 \%)$ & \\
\hline $40-49$ & 26809 & $(21.55 \%)$ & 26899 & $(21.62 \%)$ & \\
\hline $50-59$ & 23298 & $(18.72 \%)$ & 23158 & $(18.61 \%)$ & \\
\hline $60-69$ & 18124 & $(14.57 \%)$ & 18135 & $(14.58 \%)$ & \\
\hline$\geq 70$ & 16505 & $(13.27 \%)$ & 16447 & $(13.22 \%)$ & \\
\hline Gender & & & & & 0.708 \\
\hline Male & 48529 & $(39.00 \%)$ & 48438 & $(38.93 \%)$ & \\
\hline Female & 75894 & $(61.00 \%)$ & 75985 & $(61.07 \%)$ & \\
\hline Urban level & & & & & 1.000 \\
\hline Highest urbanization & 28284 & $(22.73 \%)$ & 28281 & $(22.73 \%)$ & \\
\hline High urbanization & 34946 & $(28.09 \%)$ & 34943 & $(28.08 \%)$ & \\
\hline Moderate & 21999 & $(17.68 \%)$ & 22002 & $(17.68 \%)$ & \\
\hline Low urbanization & 21939 & $(17.63 \%)$ & 21931 & $(17.63 \%)$ & \\
\hline Lowest urbanization & 17255 & $(13.87 \%)$ & 17266 & $(13.88 \%)$ & \\
\hline Income level (NT\$) & & & & & 1.000 \\
\hline$\leqq 18,780$ & 61421 & $(49.36 \%)$ & 61418 & $(49.36 \%)$ & \\
\hline $18,780-22,800$ & 33219 & $(26.70 \%)$ & 33209 & $(26.69 \%)$ & \\
\hline $22,801-28,800$ & 7347 & $(5.90 \%)$ & 7339 & $(5.90 \%)$ & \\
\hline $28,801-36,300$ & 7723 & $(6.21 \%)$ & 7719 & $(6.20 \%)$ & \\
\hline $36,301-45,800$ & 7583 & $(6.09 \%)$ & 7576 & $(6.09 \%)$ & \\
\hline$\geqq 45,800$ & 7130 & $(5.73 \%)$ & 7162 & $(5.76 \%)$ & \\
\hline
\end{tabular}

TABLE 2: The follow-up profiles between the mental illness and the control groups.

\begin{tabular}{|c|c|c|c|c|}
\hline & \multicolumn{2}{|c|}{$\begin{array}{c}\text { Mental Illness } \\
(\mathrm{N}=124,423)\end{array}$} & \multicolumn{2}{|c|}{$\begin{array}{c}\text { Control } \\
(\mathrm{N}=124,423)\end{array}$} \\
\hline The median duration of the onset of SRD (years) & 1.54 & & 3.22 & \\
\hline The mean duration of the onset of SRD (mean, SD) ${ }^{\dagger}$ & 2.12 & 2.12 & 3.28 & 2.00 \\
\hline The subsequent onset of SRD $(\mathrm{N}, \%)^{\dagger}$ & 4244 & $3.41 \%$ & 861 & $0.69 \%$ \\
\hline Alcohol-induced mental disorders & 656 & $0.53 \%$ & 68 & $0.05 \%$ \\
\hline Drug-induced mental disorders & 426 & $0.34 \%$ & 33 & $0.03 \%$ \\
\hline Alcohol dependence syndrome & 1185 & $0.95 \%$ & 159 & $0.13 \%$ \\
\hline Drug dependence & 554 & $0.45 \%$ & 23 & $0.02 \%$ \\
\hline Nondependent abuse of drugs & 2173 & $1.75 \%$ & 588 & $0.47 \%$ \\
\hline The death $(\mathrm{N}, \%)^{\dagger}$ & 10374 & $8.34 \%$ & 8583 & $6.90 \%$ \\
\hline The loss of follow-up (N, \%) & 212 & $0.17 \%$ & 197 & $0.16 \%$ \\
\hline Follow-up to the end year 2009 (N, \%) & 109593 & $88.08 \%$ & 114782 & $92.25 \%$ \\
\hline
\end{tabular}

${ }^{\dagger}$ denotes the differences in the distributions for the variables between two groups were significant $(P<0.001)$. 
TABLE 3: Estimated hazard ratio of the subsequent onset of substance-related disorders for mental illness compared to those without, adjusted for age, gender, urban level, and income level $(\mathrm{N}=248,846)$.

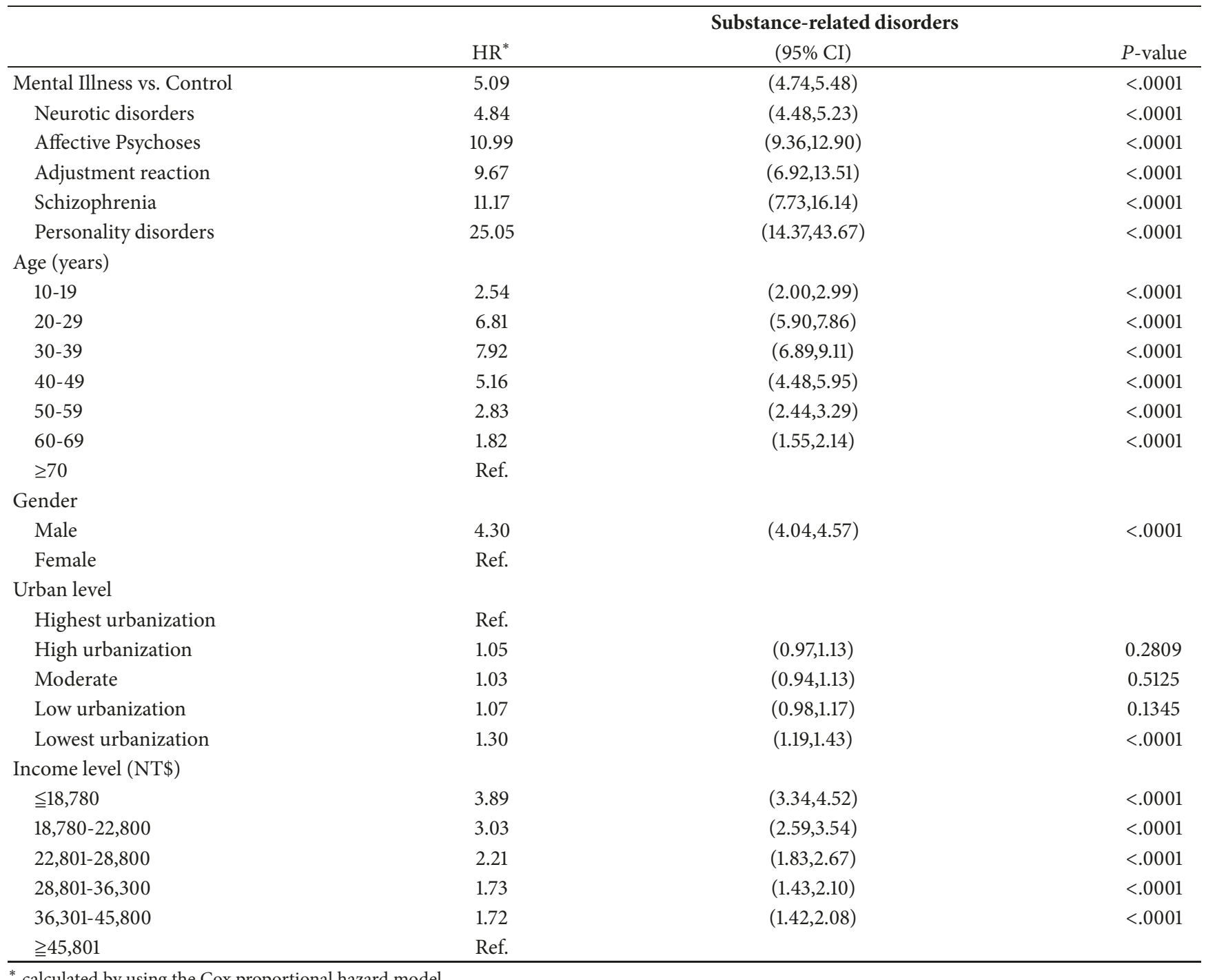

* calculated by using the Cox proportional hazard model.

gender, urban level, and income level. The results show that, for subsequently developing alcohol-induced mental disorders, the hazard ratio for affective psychoses was the highest (HRs=64.75), followed by personality disorders $(\mathrm{HR}=38.64)$, schizophrenia $(\mathrm{HR}=30.62)$, and adjustment reaction $(\mathrm{HR}=12.59)$. For subsequently developing alcohol dependence syndrome, the hazard ratio for personality disorders was the highest ( $\mathrm{HR}=47.3)$, followed by affective psychoses $(\mathrm{HR}=22.10)$, adjustment reaction $(\mathrm{HR}=21.56)$, and schizophrenia $(\mathrm{HR}=17.82)$. With regard to the risk of subsequently developing drug-related disorders, those with personality disorders had the highest risk to develop drug-induced mental disorders, drug dependence, and nondependent abuse of drugs (HRs=60.77, 82.40, and 22.63, respectively; Ps < 0.0001). Those with the second high risk of developing drug-induced mental disorders, drug dependence, and nondependent abuse of drugs were affective psychoses $(\mathrm{HR}=26.88)$, adjustment reaction $(\mathrm{HR}=68.02)$, and schizophrenia ( $\mathrm{HR}=9.37)$, respectively. However, neurotic disorders had the lowest hazard ratio of developing either alcohol-related or drug-related disorders.

\section{Discussion}

In the present study, we found that individuals with affective psychoses, neurotic disorders, schizophrenia, personality disorders, or adjustment reaction had significantly higher risks for subsequently developing SRDs compared to those without, independent of age, sex, and income and urban levels, suggesting that the prior onset of any one of the abovementioned mental disorders later on does have potential risks for developing a SRD.

Our findings are consistent with existing studies and support the onset of primary mental disorders prior to the development of substance dependence and/or abuse. Previous studies indicated that anxiety disorders, phobic 
TABLE 4: Estimated hazard ratio of the subsequent onset of substance-related disorders (mental illness vs. control) for subsamples.

\begin{tabular}{|c|c|c|c|c|}
\hline & \multicolumn{4}{|c|}{ Substance-related disorders } \\
\hline & $\mathrm{N}$ & $\mathrm{HR}^{*}$ & $(95 \% \mathrm{CI})$ & $P$-value \\
\hline \multicolumn{5}{|l|}{ Age (years) } \\
\hline $10-19$ & 10125 & 14.55 & $(7.89,26.83)$ & $<.0001$ \\
\hline $20-29$ & 29149 & 8.59 & $(7.04,10.48)$ & $<.0001$ \\
\hline $30-39$ & 40197 & 7.17 & $(6.14,8.38)$ & $<.0001$ \\
\hline $40-49$ & 53708 & 4.47 & $(3.86,5.17)$ & $<.0001$ \\
\hline $50-59$ & 46456 & 3.78 & $(3.12,4.56)$ & $<.0001$ \\
\hline $60-69$ & 36259 & 2.12 & $(1.72,2.62)$ & $<.0001$ \\
\hline$\geq 70$ & 32952 & 2.18 & $(1.65,2.87)$ & $<.0001$ \\
\hline \multicolumn{5}{|l|}{ Gender } \\
\hline Male & 96967 & 8.68 & $(7.35,10.26)$ & $<.0001$ \\
\hline Female & 151879 & 4.24 & $(3.90,4.60)$ & $<.0001$ \\
\hline \multicolumn{5}{|l|}{ Urban level } \\
\hline Highest urbanization & 56565 & 5.69 & $(4.82,6.73)$ & $<.0001$ \\
\hline High urbanization & 69889 & 5.70 & $(4.92,6.60)$ & $<.0001$ \\
\hline Moderate & 44001 & 5.29 & $(4.44,6.31)$ & $<.0001$ \\
\hline Low urbanization & 43870 & 4.93 & $(4.14,5.87)$ & $<.0001$ \\
\hline Lowest urbanization & 34521 & 3.47 & $(2.95,4.08)$ & $<.0001$ \\
\hline \multicolumn{5}{|l|}{ Income level (NT\$) } \\
\hline$\leqq 18,780$ & 122839 & 5.65 & $(5.10,6.26)$ & $<.0001$ \\
\hline $18,780-22,800$ & 66428 & 4.25 & $(3.71,4.86)$ & $<.0001$ \\
\hline $22,801-28,800$ & 14686 & 5.01 & $(3.63,6.93)$ & $<.0001$ \\
\hline $28,801-36,300$ & 15442 & 6.62 & $(4.54,9.66)$ & $<.0001$ \\
\hline $36,301-45,800$ & 15159 & 3.77 & $(2.78,5.12)$ & $<.0001$ \\
\hline$\geqq 45,801$ & 14292 & 3.45 & $(2.45,4.87)$ & $<.0001$ \\
\hline
\end{tabular}

* : subsamples were stratified by age, gender, urban level, and income level; model analyses were adjusted for other covariates.

and panic disorders, personality disorders, antisocial personality disorders, conduct disorders, mood disorders, and schizophrenia were associated with later onset of substance use disorders [5, 15-22, 24-26, 28, 36, 51-55]. Our study found that when compared to their counterparts with no specific mental disorders and regardless of the existence of other comorbid mental disorders along with their specific diseases, persons with personality disorders had the highest risk of developing a SRD $(\mathrm{HR}=25.05)$, followed by schizophrenia $(\mathrm{HR}=11.17)$, affective psychoses $(\mathrm{HR}=10.99)$, adjustment reaction $(\mathrm{HR}=9.67)$, and neurotic disorders $(\mathrm{HR}=4.84)$. And this order was the same as that found for developing nondependent abuse of drugs-the major subtype in the SRD onsets.

In subsample analyses, we found that, among all age groups, the youngest group of 10-19 years is at the highest risk for those with any specific mental disorders to develop a SRD in their later life. Review articles pinpointed that child psychopathology is associated with early onset of substance use and abuse in later adolescence $[56,57]$. In addition, US epidemiological studies showed that mental disorders usually occur at an earlier age than do substance use disorders, and the highest risk of secondary substance use disorders was found among people whose mental disorders begin during either childhood or adolescence [58], and the median time interval between first onset of primary mental disorders and first onset of secondary substance disorders is 5 years or more [58]. A study for the National Institute of Mental Health (NIMH) epidemiologic Catchment Area Program reported that the lifetime prevalence of onset sequencing pattern for any mood disorder occurring first is higher than for any substance use disorder, and at least $75 \%$ of comorbid subjects had their first onset disorder before age 20 [26]. In addition, one thing that should be noted is that attentiondeficit/hyperactivity disorder (ADHD) in childhood and adolescence has been suggested to be related to subsequent SRD in adulthood $[59,60]$. The shortage of subjects aged 1019 years in this study along with the low reported prevalence rates of ADHD in Taiwan [61], however, led us not to include it in our analyses. Nevertheless, the risk of ADHD for developing a SRD is worth examining in a future study.

By controlling for the studied mental disorders and other confounding variables, we found the risks for developing a SRD were over five times higher for people aged 20-49 years and over two times higher for people aged 10-19 or 50-59 years, compared to the risk for people aged 70 years and older. As the mean duration of the onset of SRDs for people with the specific mental disorders was 2.12 years $(\mathrm{SD}=2.12)$, which was significantly $(p<0.001)$ less than 3.28 years $(\mathrm{SD}=2.00)$ in people without (median duration: 1.54 and 3.22 years, respectively), we suggest that health practitioners should pay 
TABLE 5: Estimated hazard ratio of the subsequent onset of each subtype of SRDs for those with the selected mental disorders compared to those without, adjusted for age, gender, urban, and income levels.

\begin{tabular}{|c|c|c|c|}
\hline $\begin{array}{l}\text { Dependent variable } \\
\text { Independent variable }\end{array}$ & HR & $(95 \% \mathrm{CI})$ & $p$-value \\
\hline \multicolumn{4}{|c|}{ Alcohol-induced mental disorders } \\
\hline Neurotic disorders & 8.75 & $(6.78-11.29)$ & $<.0001$ \\
\hline Affective psychoses & 64.75 & $(26.76-156.68)$ & $<.0001$ \\
\hline Adjustment reaction & 12.59 & $(3.88-40.90)$ & $<.0001$ \\
\hline Schizophrenia & 30.62 & $(7.48-125.31)$ & $<.0001$ \\
\hline Personality disorders & 38.64 & $(5.29-282.15)$ & 0.0003 \\
\hline \multicolumn{4}{|c|}{ Drug-induced mental disorders } \\
\hline Neurotic disorders & 11.47 & $(7.99-14.47)$ & $<.0001$ \\
\hline Affective psychoses & 26.88 & $(13.27-54.48)$ & $<.0001$ \\
\hline Adjustment reaction & 14.39 & $(4.47-46.40)$ & $<.0001$ \\
\hline Schizophrenia & 22.51 & $(7.07-71.65)$ & $<.0001$ \\
\hline Personality disorders & 60.77 & $(8.41-439.17)$ & $<.0001$ \\
\hline \multicolumn{4}{|c|}{ Alcohol dependence syndrome } \\
\hline Neurotic disorders & 6.98 & $(5.89-8.29)$ & $<.0001$ \\
\hline Affective psychoses & 22.1 & $(14.91-32.75)$ & $<.0001$ \\
\hline Adjustment reaction & 21.56 & $(7.91-58.82)$ & $<.0001$ \\
\hline Schizophrenia & 17.82 & $(7.23-43.91)$ & $<.0001$ \\
\hline Personality disorders & 47.3 & $(11.64-192.22)$ & $<.0001$ \\
\hline \multicolumn{4}{|l|}{ Drug dependence } \\
\hline Neurotic disorders & 25.32 & $(16.19-39.60)$ & $<.0001$ \\
\hline Affective psychoses & 47.25 & $(21.05-106.08)$ & $<.0001$ \\
\hline Adjustment reaction & 68.02 & $(9.44-490.12)$ & $<.0001$ \\
\hline Schizophrenia & 64.71 & $(8.97-466.70)$ & $<.0001$ \\
\hline Personality disorders & 82.4 & $(11.45-592.81)$ & $<.0001$ \\
\hline \multicolumn{4}{|c|}{ Nondependent abuse of drugs } \\
\hline Neurotic disorders & 3.75 & $(3.41-4.13)$ & $<.0001$ \\
\hline Affective psychoses & 7.07 & $(5.83-8.58)$ & $<.0001$ \\
\hline Personality disorders & 6.07 & $(4.12-8.94)$ & $<.0001$ \\
\hline Schizophrenia & 9.37 & $(5.83-15.06)$ & $<.0001$ \\
\hline Personality disorders & 22.63 & $(10.59-48.35)$ & $<.0001$ \\
\hline
\end{tabular}

more attention to the possible use of substances with their clients who have mental problems and adopt some strategies for early prevention or prompt treatment.

In our study, males were more likely to develop SRDs than females and the risk was about 4 times higher. This gender difference in substance use or disorders has been found across different age groups and nations $[16,18,19,62-$ 64]. Nevertheless, there may exist gender differences in the magnitudes of the risks for developing specific substance use disorders according to different mental disorders $[65,66]$. Our results also revealed that the risk of developing a SRD for people living in the lowest urbanization area is 30\% higher than the risk for people living in the highest urbanizationlevel area. This finding is consistent with the findings of Simmons and Havens' study (2007) [46]. We also found that people in lower income levels were at higher risks for developing SRDs, and the risk increased as the income level declined.

Our study found that affective psychoses and personality disorders were at greatest risk for developing alcohol-related disorders. The likelihood of developing alcohol dependence syndrome and drug-related disorders (drug-induced mental disorders, drug dependence, and nondependent abuse of drugs) were also particularly high for patients with personality disorders. Moreover, the high hazard ratios of developing drug dependence for patients with adjustment reaction and of developing nondependent abuse of drugs for patients with schizophrenia are notable. However, these findings suggest an important thing; that is, health professionals in clinical settings should take steps to prevent patients with personality disorders from substance use and abuse although the prevalence of the disorders is not as high as those of major mental disorders such as affective psychoses and schizophrenia.

In addition, the variation in risk for each subtype of substance-related disorders across mental disorders shown in our study results suggests a need for future studies to investigate the relative risks across a broader range of mental disorders for each category of SRDs. Such studies would provide information for different etiological pathways and tailored prevention strategies. 
Our study has several strengths. First, to our knowledge, of all the studies on the relationship between mental disorders and SRDs, this study is the one with the largest sample size and study subjects covering individuals in late childhood, adolescence, adulthood, and old age. Second, we excluded subjects with SRDs before the index date, on the index date, and one year after the index date. This was done to ascertain that no subjects had any SRDs prior to the onsets of mental disorders in order to examine the concerned temporal link between the mental disorders of interest and SRDs.

There are some limitations in the present study. First, there is a lack of some information on related biomarkers such as genetic data, body mass index, hormones, education, lifestyles (e.g., physical activity, smoking, drinking, and drug use or abuse), sleep quality, and family medical history (e.g., psychiatric disorders, substance use/abuse). Second, it is impossible to clarify the severity of the diseases among the cases from the data since the cases were identified by ICD-9$\mathrm{CM}$ codes. To ensure the accuracy in identifying actual cases, we restricted the diagnosis of disease to three outpatient visits within one year or a one-time hospitalization. Third, we were unable to access the patient's living address from our database due to Personal Data Protection Law and the data actually provided us the local areas in which beneficiaries enrolled in the health insurance program via their employment systems and so these areas may not be their residing but working areas. However, despite the above-mentioned weaknesses in this study, the strengths of the NHIRD with longitudinal data since 1995 provide a good opportunity for us to establish a temporal relationship between the specific mental disorders and SRDs.

In the present study, we provided evidence on the temporal link between the specific mental disorders and substancerelated disorders in which a prior onset of any selected mental disorders could increase the risks for developing SRDs. Due to the limitation of the sampling design in NHIRD, we were unable to trace the disease trajectory for each subject for an entire birth cohort. Therefore, we caution against assuming a causal relationship between these two classified disorders.

\section{Conclusion}

In this population-based cohort study, we found that individuals with selected mental disorders, including affective psychoses, neurotic disorders, personality disorders, and adjustment reaction, had a fivefold higher risk for subsequent onset of substance-related disorders as compared to those without selected mental disorders, and among them, those with personality disorders had the highest risk. The duration from observing a mental disorder to having a later onset of substance-related disorder is around two years, suggesting that health professionals in their practices should pay more attention to the individuals with mental problems from substance use and abuse.

\section{Abbreviations}

SRDs: Substance-Related Disorders

SUDs: Substance Use Disorders
SIDs: Substance-Induced

Disorders

UNODC: The United Nations Office on Drugs and Crime

NHIRD: The National Health

Insurance Research

Database

TNHIP: The Taiwan National Health Insurance Program

CCHIA: The Collaboration Center of Health Information Application

NIMH: The National Institute of Mental Health.

\section{Data Availability}

The data are not available because of confidentiality and restriction by law from Ministry of Health and Welfare, Taiwan.

\section{Disclosure}

The institute had no role in study design, data collection and analysis, decision to publish, or preparation of the manuscript. This paper is based on part of Ph.D. dissertation of the first Dr. Mu-Lin Chiu and has been presented as an abstract at "7th International Conference on Addictive Disorders, Addiction Medicine and Pharmaceuticals" and at "4th International Conference and Exhibition on Addiction Research \& Therapy”.

\section{Conflicts of Interest}

We declare that we have no conflicts of interest.

\section{Authors' Contributions}

Trong-Neng Wu had full access to all of the data in the study and was responsible for the integrity of the data and study interpretation. Chiu-Ying Chen and Trong-Neng Wu had the idea for and designed the study and was responsible for the accuracy of the data analysis and study interpretation. ChiuYing Chen and Trong-Neng Wu have equal contribution as corresponding authors. Mu-Lin Chiu, Chi-Fung Cheng, Wen-Miin Liang, and Chiu-Ying Chen analyzed and interpreted the data. Mu-Lin Chiu and Chiu-Ying Chen drafted the article and submitted the paper for publication. ChiuYing Chen and $\mathrm{Mu}$-Lin Chiu have equal contribution as first authors. Mu-Lin Chiu, Chiu-Ying Chen, Pen-Tang Lin, and Trong-Neng Wu critically revised the article for important intellectual contents. Trong-Neng $\mathrm{Wu}$, Chiu-Ying Chen, $\mathrm{Mu}-$ Lin Chiu, Chi-Fung Cheng, Wen-Miin Liang, and Pen-Tang Lin provided administrative, technical, logistical, or material support. All authors had the final approval of the article. Chiu-Ying Chen and Trong-Neng Wu contributed equally as corresponding authors. 


\section{Acknowledgments}

All authors would like to thank the Collaboration Center of Health Information Application (CCHIA), Ministry of Health and Welfare of Taiwan for providing the NHI database.

\section{References}

[1] UNODC, World Drug Report 2012, United Nations, New York, NY, USA, 2012.

[2] L. Degenhardt, H. Whiteford, and W. D. Hall, "The Global Burden of Disease projects: What have we learned about illicit drug use and dependence and their contribution to the global burden of disease?" Drug and Alcohol Review, vol. 33, no. 1, pp. 4-12, 2014.

[3] INCB, Economic consequences of drug abuse, International Narcotics Control Board, Austria, 2013.

[4] D. A. Regier, M. E. Farmer, D. S. Rae et al., "Comorbidity of mental disorders with alcohol and other drug abuse. Results from the epidemiologic catchment area (ECA) study," The Journal of the American Medical Association, vol. 264, no. 19, pp. 2511-2518, 1990.

[5] E. Asselmann, H.-U. Wittchen, R. Lieb, M. Höfler, and K. Beesdo-Baum, "Associations of fearful spells and panic attacks with incident anxiety, depressive, and substance use disorders: A 10-year prospective-longitudinal community study of adolescents and young adults," Journal of Psychiatric Research, vol. 55, no. 1, pp. 8-14, 2014.

[6] R. C. Kessler, “The epidemiology of dual diagnosis," Biological Psychiatry, vol. 56, no. 10, pp. 730-737, 2004.

[7] V. Agosti, E. Nunes, and F. Levin, "Rates of psychiatric comorbidity among U.S. residents with lifetime cannabis dependence," American Journal of Drug and Alcohol Abuse, vol. 28, no. 4, pp. 643-652, 2002.

[8] A. Kenneson, J. S. Funderburk, and S. A. Maisto, "Substance use disorders increase the odds of subsequent mood disorders," Drug and Alcohol Dependence, vol. 133, no. 2, pp. 338-343, 2013.

[9] D. M. Fergusson, J. M. Boden, and L. J. Horwood, "Tests of causal links between alcohol abuse or dependence and major depression," Archives of General Psychiatry, vol. 66, no. 3, pp. 260-266, 2009.

[10] J. M. Boden, D. M. Fergusson, and L. J. Horwood, "Cigarette smoking and depression: Tests of causal linkages using a longitudinal birth cohort," The British Journal of Psychiatry, vol. 196, no. 6, pp. 440-446, 2010.

[11] M. R. Hayatbakhsh, J. M. Najman, K. Jamrozik, A. A. Mamun, R. Alati, and W. Bor, "Cannabis and anxiety and depression in young adults: A large prospective study," Journal of the American Academy of Child and Adolescent Psychiatry, vol. 46, no. 3, pp. 408-417, 2007.

[12] O. Klungsøyr, J. F. Nygård, T. Sørensen, and I. Sandanger, "Cigarette smoking and incidence of first depressive episode: An 11-year, population-based follow-up study," American Journal of Epidemiology, vol. 163, no. 5, pp. 421-432, 2006.

[13] M. van Laar, S. van Dorsselaer, K. Monshouwer, and R. de Graaf, "Does cannabis use predict the first incidence of mood and anxiety disorders in the adult population?" Addiction, vol. 102, no. 8, pp. 1251-1260, 2007.
[14] L. Boschloo, N. Vogelzangs, W. Van Den Brink et al., "Alcohol use disorders and the course of depressive and anxiety disorders," The British Journal of Psychiatry, vol. 200, no. 6, pp. 476484, 2012.

[15] J. D. Buckner, N. B. Schmidt, A. R. Lang, J. W. Small, R. C. Schlauch, and P. M. Lewinsohn, "Specificity of social anxiety disorder as a risk factor for alcohol and cannabis dependence," Journal of Psychiatric Research, vol. 42, no. 3, pp. 230-239, 2008.

[16] P.-H. Kuo, C. O. Gardner Jr., K. S. Kendler, and C. A. Prescott, "The temporal relationship of the onsets of alcohol dependence and major depression: using a genetically informative study design,” Psychological Medicine, vol. 36, no. 8, pp. 1153-1162, 2006.

[17] J. Sareen, M. Chartier, M. P. Paulus, and M. B. Stein, "Illicit drug use and anxiety disorders: Findings from two community surveys," Psychiatry Research, vol. 142, no. 1, pp. 11-17, 2006.

[18] L. Degenhardt, W. T. Chiu, K. Conway et al., "Does the gateway matter? Associations between the order of drug use initiation and the development of drug dependence in the National Comorbidity Study Replication," Psychological Medicine, vol. 39, no. 1, pp. 157-167, 2009.

[19] J. Wang and S. B. Patten, "A Prospective Study of Sex-Specific Effects of Major Depression on Alcohol Consumption," The Canadian Journal of Psychiatry, vol. 46, no. 5, pp. 422-425, 2016.

[20] M. R. Crone and S. A. Reijneveld, "The association of behavioural and emotional problems with tobacco use in adolescence," Addictive Behaviors, vol. 32, no. 8, pp. 1692-1698, 2007.

[21] A. Kenneson, J. S. Funderburk, and S. A. Maisto, "Risk factors for secondary substance use disorders in people with childhood and adolescent-onset bipolar disorder: Opportunities for prevention," Comprehensive Psychiatry, vol. 54, no. 5, pp. 439-446, 2013.

[22] P. Zimmermann, H.-U. Wittchen, M. Höfler, H. Pfister, R. C. Kessler, and R. Lieb, "Primary anxiety disorders and the development of subsequent alcohol use disorders: A 4-year community study of adolescents and young adults," Psychological Medicine, vol. 33, no. 7, pp. 1211-1222, 2003.

[23] S. M. Strakowski and M. P. Delbello, "The co-occurrence of bipolar and substance use disorders," Clinical Psychology Review, vol. 20, no. 2, pp. 191-206, 2000.

[24] P. Cohen, H. Chen, T. N. Crawford, J. S. Brook, and K. Gordon, "Personality disorders in early adolescence and the development of later substance use disorders in the general population," Drug and Alcohol Dependence, vol. 88, no. 1, pp. S71-S84, 2007.

[25] M. H. Boyle, D. R. Offord, Y. A. Racine, P. Szatmari, J. E. Fleming, and P. S. Links, "Predicting substance use in late adolescence: Results from the Ontario Child Health Study follow-up," The American Journal of Psychiatry, vol. 149, no. 6, pp. 761-767, 1992.

[26] K. A. Christie, J. D. Burke Jr., D. A. Regier, D. S. Rae, J. H. Boyd, and B. Z. Locke, "Epidemiologic evidence for early onset of mental disorders and higher risk of drug abuse in young adults," The American Journal of Psychiatry, vol. 145, no. 8, pp. 971-975, 1988.

[27] R. D. Goodwin, D. M. Fergusson, and L. J. Horwood, "Association between anxiety disorders and substance use disorders among young persons: Results of a 21-year longitudinal study," Journal of Psychiatric Research, vol. 38, no. 3, pp. 295-304, 2004.

[28] M. Fatséas, C. Denis, E. Lavie, and M. Auriacombe, "Relationship between anxiety disorders and opiate dependence- A 
systematic review of the literature. Implications for diagnosis and treatment," Journal of Substance Abuse Treatment, vol. 38, no. 3, pp. 220-230, 2010.

[29] D. E. Ramo, K. L. Delucchi, S. M. Hall, H. Liu, and J. J. Prochaska, "Marijuana and tobacco co-use in young adults: Patterns and thoughts about use," Journal of Studies on Alcohol and Drugs, vol. 74, no. 2, pp. 301-310, 2013.

[30] L. Atwoli, P. A. Mungla, M. N. Ndung'u, K. C. Kinoti, and E. M. Ogot, "Prevalence of substance use among college students in Eldoret, western Kenya," BMC Psychiatry, vol. 11, article 34, 2011.

[31] M. S. O'Brien and J. C. Anthony, "Risk of becoming cocaine dependent: Epidemiological estimates for the United States, 2000-2001," Neuropsychopharmacology, vol. 30, no. 5, pp. 10061018, 2005.

[32] R. E. Isralowitz and A. Peleg, "Israeli college student alcohol use: The association of background characteristics and regular drinking patterns," Drug and Alcohol Dependence, vol. 42, no. 3, pp. 147-153, 1996.

[33] M. Baumann, E. Spitz, F. Guillemin et al., "Associations of social and material deprivation with tobacco, alcohol, and psychotropic drug use, and gender: a population-based study," International Journal of Health Geographics, vol. 6, article 50, 2007.

[34] C. E. Grella, "Effects of gender and diagnosis on addiction history, treatment utilization, and psychosocial functioning among a dually-diagnosed sample in drug treatment," Journal of Psychoactive Drugs, vol. 35, pp. 169-179, 2003.

[35] J. Du, D. Huang, M. Zhao, and Y. I. Hser, "Drug-abusing offenders with co-morbid mental disorders: gender differences in problem severity, treatment participation, and recidivism," Biomedical and Environmental Sciences, vol. 26, no. 1, pp. 32-39, 2013.

[36] K. P. Kushwaha, Y. D. Singh, A. K. Rathi, K. P. Singh, and C. K. Rastogi, "Prevalence and abuse of psychoactive substances in children and adolescents," The Indian Journal of Pediatrics, vol. 59, no. 2, pp. 261-268, 1992.

[37] C. J. Conover, P. Arno, M. Weaver, A. Ang, and S. L. Ettner, "Income and employment of people living with combined HIV/AIDS, chronic mental illness, and substance abuse disorders," The Journal of Mental Health Policy and Economics, vol. 9, no. 2, pp. 71-86, 2006.

[38] S. W. Hwang, M. M. Agha, M. I. Creatore, and R. H. Glazier, "Age- and sex-specific income gradients in alcohol-related hospitalization rates in an urban area," Annals of Epidemiology, vol. 15, no. 1, pp. 56-63, 2005.

[39] R. D. Goodwin, J. Pagura, R. Spiwak, A. R. Lemeshow, and J. Sareen, "Predictors of persistent nicotine dependence among adults in the United States," Drug and Alcohol Dependence, vol. 118, no. 2-3, pp. 127-133, 2011.

[40] M. Amiri, M. Dejman, M. Dastoury, and P. Roushanfekr, "The relationship between addiction and socio-demographic characteristics of Iranian newcomer prisoners.," Global Journal of Health Science, vol. 6, no. 2, pp. 168-174, 2014.

[41] J. C. Mwenifumbo, E. M. Sellers, and R. F. Tyndale, "Socioeconomic and drug use determinants of smoking status in an urban adult population of Black African descent," Nicotine \& Tobacco Research, vol. 10, no. 8, pp. 1319-1325, 2008.

[42] L. De Saxe Zerden, L. M. Lundgren, D. Chassler, A. C. Horowitz, E. Adorno, and T. Purington, "Social and economic factors associated with recent and lifetime incarceration among Puerto
Rican drug users," Journal of Ethnicity in Substance Abuse, vol. 12, no. 2, pp. 179-195, 2013.

[43] K. Gauffin, B. Vinnerljung, M. Fridell, M. Hesse, and A. Hjern, "Childhood socio-economic status, school failure and drug abuse: A Swedish national cohort study," Addiction, vol. 108, no. 8, pp. 1441-1449, 2013.

[44] L. Bowes, A. Chollet, E. Fombonne, C. Galéra, and M. Melchior, "Lifecourse SEP and tobacco and cannabis use," European Journal of Public Health, vol. 23, no. 2, pp. 322-327, 2013.

[45] K. J. Neufeld, D. H. Peters, M. Rani, S. Bonu, and R. K. Brooner, "Regular use of alcohol and tobacco in India and its association with age, gender, and poverty," Drug and Alcohol Dependence, vol. 77, no. 3, pp. 283-291, 2005.

[46] L. A. Simmons and J. R. Havens, "Comorbid substance and mental disorders among rural Americans: Results from the national comorbidity survey," Journal of Affective Disorders, vol. 99, no. 1-3, pp. 265-271, 2007.

[47] K. Peltzer, A. Davids, and P. Njuho, "Alcohol use and problem drinking in South Africa: findings from a national populationbased survey," African Journal of Psychiatry, vol. 14, no. 1, pp. 30-37, 2011.

[48] P. M. O’Malley, L. D. Johnston, and J. G. Bachman, "Cocaine use among American adolescents and young adults," NIDA Research Monograph, vol. 61, pp. 50-75, 1985.

[49] Ministry of Health and Welfare, "National Health Insurance Annual Report 2014-2015, Tech. Rep., Taiwan, 2014.

[50] C. Y. Liu, Y. T. Hung, and Y. L. Chuang, "Incorporating development stratification of Taiwan townships into sampling design of large scale health interview survey," Journal of Health Management, vol. 4, no. 1, pp. 1-22, 2006.

[51] C. B. Pedersen, O. Mors, A. Bertelsen et al., "A comprehensive nationwide study of the incidence rate and lifetime risk for treated mental disorders," JAMA Psychiatry, vol. 71, no. 5, pp. 573-581, 2014.

[52] J. B. Kaplow, P. J. Curran, A. Angola, and E. Jane Costello, “The Prospective Relation between Dimensions of Anxiety and the Initiation of Adolescent Alcohol Use," Journal of Clinical Child \& Adolescent Psychology, vol. 30, no. 3, pp. 316-326, 2001.

[53] J. Mendlewicz and M. Baron, "Morbidity risks in subtypes of unipolar depressive illness: Differences between early and late onset forms," The British Journal of Psychiatry, vol. 139, no. 5, pp. 463-466, 1981.

[54] M. Hambrecht and H. Häfner, "Substance abuse and the onset of schizophrenia," Biological Psychiatry, vol. 40, no. 11, pp. 11551163, 1996.

[55] P. Central, "Schizophrenia Symptoms," 2016, http://psychcentral.com/disorders/schizophrenia-symptoms/.

[56] T. D. Armstrong and E. J. Costello, "Community studies on adolescent substance use, abuse, or dependence and psychiatric comorbidity," Journal of Consulting and Clinical Psychology, vol. 70, no. 6, pp. 1224-1239, 2002.

[57] C. Couwenbergh, W. Van Den Brink, K. Zwart, C. Vreugdenhil, P. Van Wijngaarden-Cremers, and R. J. Van Der Gaag, "Comorbid psychopathology in adolescents and young adults treated for substance use disorders: A review," European Child and Adolescent Psychiatry, vol. 15, no. 6, pp. 319-328, 2006.

[58] R. A.-G. S. Kessler, L. Andrade, R. Bijl et al., Cross-national comparisons of comorbidities between substance use disorders and mental disorders: Results from the International Consortium in Psychiatric Epidemiology, Plenum Publishers, New York, NY, USA, 2003. 
[59] C. A. Zulauf, S. E. Sprich, S. A. Safren, and T. E. Wilens, "The Complicated Relationship Between Attention Deficit/ Hyperactivity Disorder and Substance Use Disorders," Current Psychiatry Reports, vol. 16, no. 3, 2014.

[60] A. P. Groenman, J. Oosterlaan, N. Rommelse et al., "Substance use disorders in adolescents with attention deficit hyperactivity disorder: A 4-year follow-up study," Addiction, vol. 108, no. 8, pp. 1503-1511, 2013.

[61] L. Wang, S. Lee, S. Yuan et al., "Prevalence rates of youths diagnosed with and medicated for ADHD in a nationwide survey in Taiwan from 2000 to 2011," Epidemiology and Psychiatric Sciences, vol. 26, no. 06, pp. 624-634, 2017.

[62] B. Bühler, M. Hambrecht, W. Löffler, W. An Der Heiden, and H. Häfner, "Precipitation and determination of the onset and course of schizophrenia by substance abuse - A retrospective and prospective study of 232 population-based first illness episodes," Schizophrenia Research, vol. 54, no. 3, pp. 243-251, 2002.

[63] B. F. Grant and T. C. Harford, "Comorbidity between DSM-IV alcohol use disorders and major depression: results of a national survey," Drug and Alcohol Dependence, vol. 39, no. 3, pp. 197206, 1995.

[64] D. G. Blazer and L. Wu, "The Epidemiology of Substance Use and Disorders Among Middle Aged and Elderly Community Adults: National Survey on Drug Use and Health," The American Journal of Geriatric Psychiatry, vol. 17, no. 3, pp. 237-245, 2009.

[65] C. B. Fleming, W. A. Mason, J. J. Mazza, R. D. Abbott, and R. F. Catalano, "Latent growth modeling of the relationship between depressive symptoms and substance use during adolescence.," Psychology of Addictive Behaviors, vol. 22, no. 2, pp. 186-197, 2008.

[66] J. Wang and S. B. Patten, "Prospective study of frequent heavy alcohol use and the risk of major depression in the Canadian general population," Depression and Anxiety, vol. 15, no. 1, pp. $42-45,2002$. 


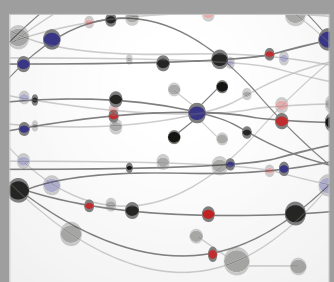

The Scientific World Journal
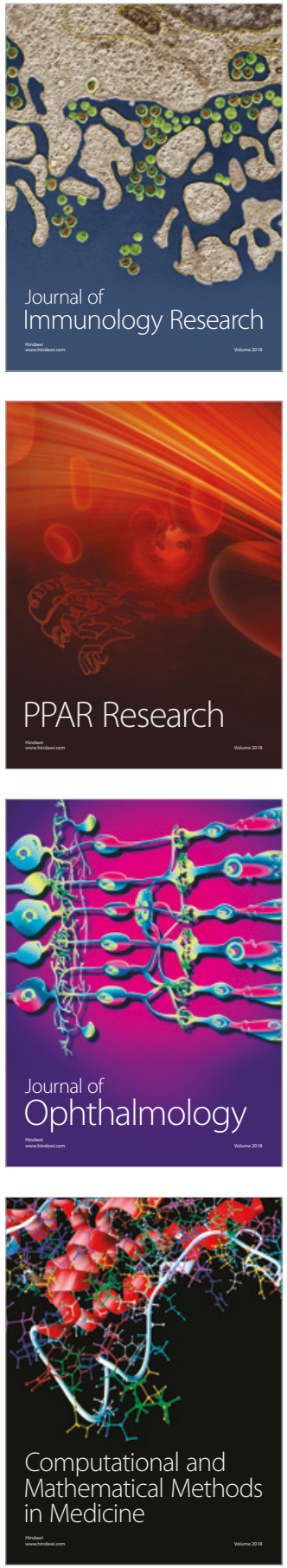

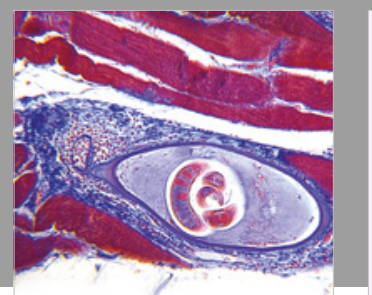

Gastroenterology Research and Practice

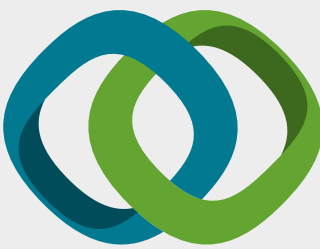

\section{Hindawi}

Submit your manuscripts at

www.hindawi.com
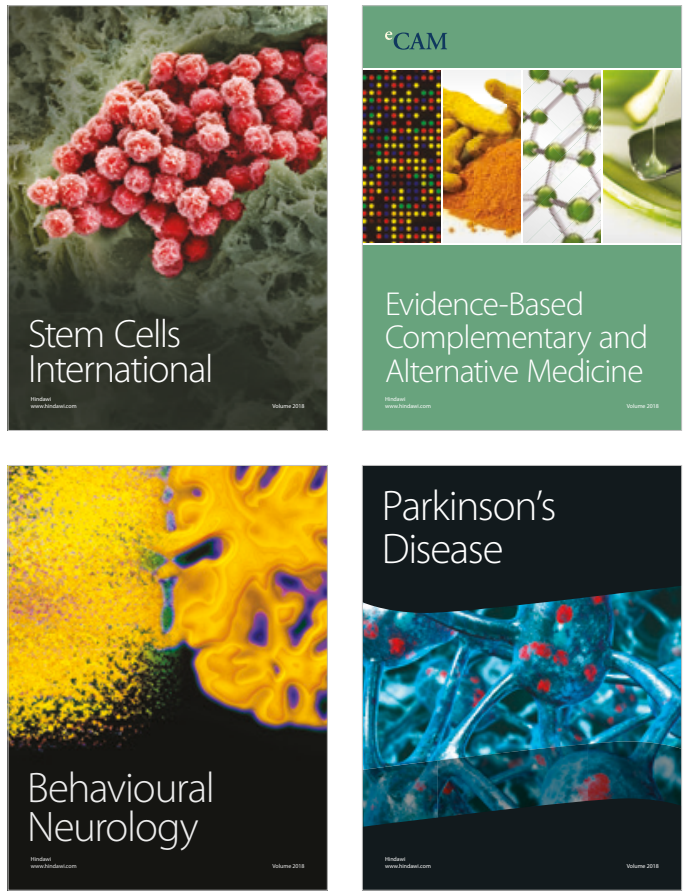

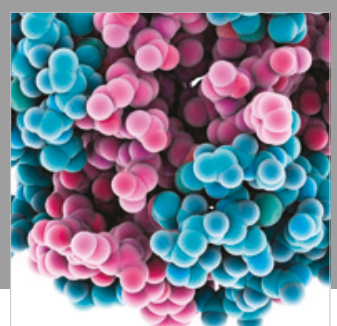

ournal of

Diabetes Research

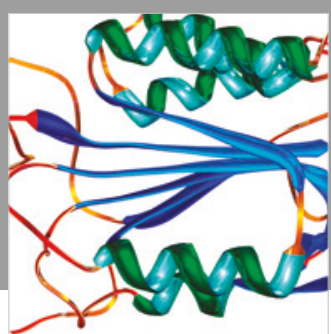

Disease Markers
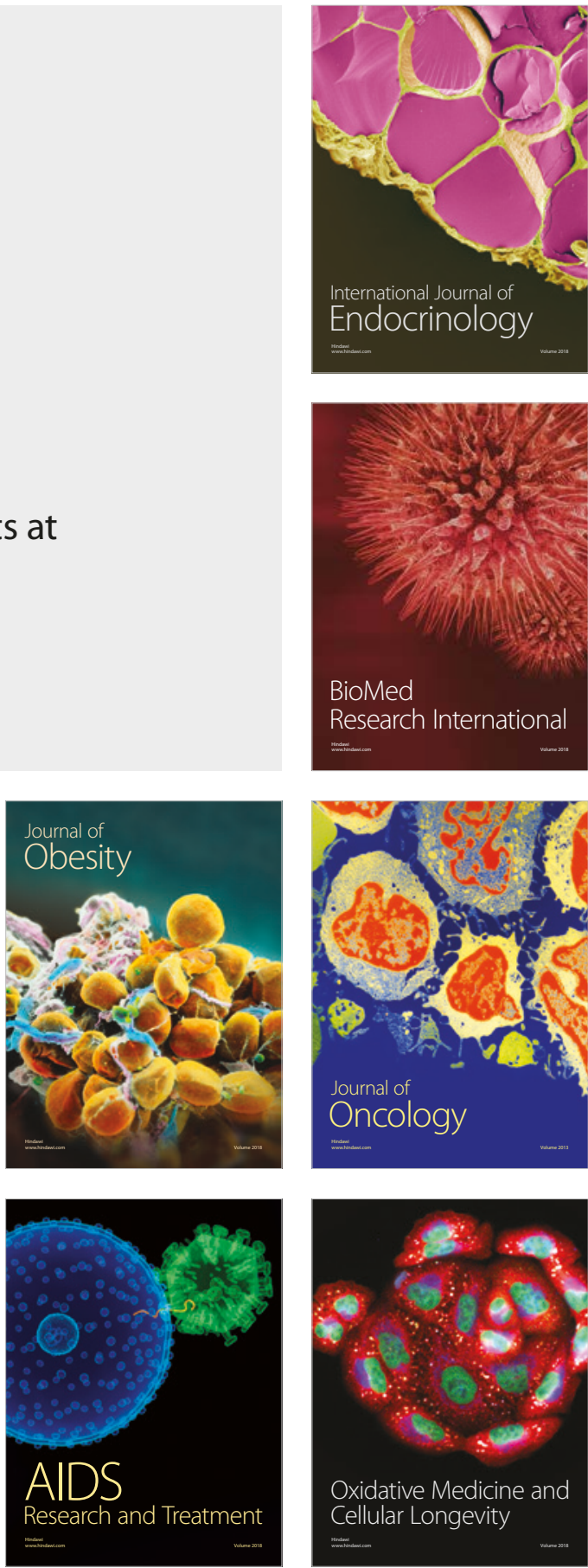